\section{POS0863 DIFFERENTIATING THE DOMINANCE OF PULMONARY VASCULAR DISEASE OR INTERSTITIAL LUNG DISEASE ON HEMODYNAMIC ABNORMALITIES IN SYSTEMIC SCLEROSIS AND CLARIFYING EACH CHARACTERISTIC BY USING QUANTITATIVE EVALUATION OF CHEST CT}

K. Ninagawa ${ }^{1}$, M. Kato ${ }^{1}$, M. Kono ${ }^{1}$, Y. Fujieda ${ }^{1}$, K. Oku ${ }^{1}$, T. Atsumi ${ }^{1} .{ }^{1}$ Hokkaido University, Department of Rheumatology, Endocrinology and Nephrology, Faculty of Medicine and Graduate School of Medicine, Sapporo, Japan

Background: Group 1 and 3 pulmonary hypertension $(\mathrm{PH})$ develop through different pathological mechanisms but have similar hemodynamic abnormalities. Systemic sclerosis (SSc) is associated with both pulmonary vascular disease (PVD) and interstitial lung disease (ILD), making it challenging to differentiate group 1 and $3 \mathrm{PH}$ in those patients. A previous study using quantitative evaluation of chest computed tomography (CT) demonstrated that normal lung volume was inversely correlated with mean pulmonary arterial pressure (mPAP) in patients with group $3 \mathrm{PH}(1)$.

Objectives: In this study, we aimed to assess the dominance of PVD or ILD in SSc patients by quantitative evaluation of chest CT and to evaluate each characteristic.

Methods: A total of 76 SSc patients who underwent right heart catheterization (RHC) were included. Chest CT was evaluated by using a software (Synapse Vincent Ver.3.0, Fujifilm) which quantified normal and total area of the lung. Then, we calculated abnormal area by drawing normal area from total area in the lung (\%). Pulmonary function test (PFT) and serum biomarkers, such as $\mathrm{KL}-6$ and $\mathrm{LDH}$, were also evaluated. The dominance of PVD or ILD was defined as divergent or parallel change between the first and last assessments, respectively, in mPAP and abnormal area in the lung calculated using the software. Increase or decrease by over $10 \%$ in the last assessment compared to the first assessment was considered as a significant change in mPAP or abnormal area in the lung. P values were calculated by Mann-Whitney $\mathrm{U}$ test, and correlation coefficients were calculated by direct regression variance.

Results: The median [range] values of mPAP and abnormal area in the lung at baseline were 23 [9-65] mmHg and 30.2 [0-100] \%, respectively. Of $37 \mathrm{SSc}$ and PH patients, 18 were defined as having PVD dominance while 19 as ILD dominance. Abnormal area in the lung at baseline was greater in patients with ILD dominance compared to those with PVD dominance (39.1 [16.3-98.3] v.s. $14.0[0-99] \%, p=0.002$ ), whereas mPAP was higher in patients with PVD dominance than those with ILD dominance (42.5 [23.0-65.0] v.s. 26.0 [16.0-42.0] $\mathrm{mmHg}, \mathrm{p}=0.002$ ). PFT parameters including forced vital capacity were not different between the two groups. The ratio of mPAP/KL- 6 showed a great difference between the two groups with its significant elevation in patients with PVD dominance $(p=0.007)$.

Conclusion: Quantitative evaluation of chest CT showed great efficiency in differentiating the dominance of PVD or ILD in patients with SSc and PH. In addition, the ratio of $\mathrm{mPAP} / \mathrm{KL}-6$ may easily be used as a parameter for dominance evaluation.

REFERENCES

[1] Iwasawa T, Kato S, Ogura T, Kusakawa Y, Iso S, Baba T, et al. Low-normal lung volume correlates with pulmonary hypertension in fibrotic idiopathic interstitial pneumonia: computer-aided 3D quantitative analysis of chest CT. AJR Am J Roentgenol. 2014;203(2):W166-73.

Disclosure of Interests: None declared

DOI: 10.1136/annrheumdis-2021-eular.2569

\section{POS0864 \\ ELIGIBILITY FOR ANTI-FIBROTIC TREATMENT WITH NINTEDANIB OF PATIENTS WITH SYSTEMIC SCLEROSIS RELATED INTERSTITIAL LUNG DISEASE}

E. De Lorenzis ${ }^{1}$, G. Natalello ${ }^{1}$, L. Verardi ${ }^{1}$, L. Gigante ${ }^{1}$, M. A. D'agostino ${ }^{1}$, S. L. Bosello'. ${ }^{1}$ Fondazione Policlinico Universitario A. Gemelli IRCCS - Catholic University of the Sacred Heart, Rheumatology Unit, Rome, Italy

Background: Pulmonary involvement is the main determinant of disease-related mortality in systemic sclerosis (SSc). The SENSCIS and INBUILD trials have documented the efficacy of the antifibrotic drug Nintedanib (NTN) in the treatment of SSc-related interstitial lung disease (SSc-ILD) and progressive forms of ILD other than Idiopathic Pulmonary Fibrosis ${ }^{1,2}$. The criteria for the use of this drug in patients with SSc-ILD in clinical practice are currently being defined. Objectives: To evaluate the proportion of patients eligible for NTN treatment based on the enrollment criteria fof the SENSCIS and INBUILD studies in a reallife cohort of SSc-ILD patients.

Methods: We considered consecutive patients with ILD extension on CT $\geq 10 \%$ and disease duration $\leq 7$ years with at least one visit in the period between September 2016 and December 2019. Data of the clinical visits were retrospectively evaluated. For each patient, we examined the visits during which a well-tolerated immunosuppressive therapy was modified because of progression of SSc-ILD and the most recent follow-up visit. Eligibility of patients for NTN was defined according to the inclusion criteria of the SENSCIS and INBUILD trials. Patients with more than 2 acral ulcers at the time of evaluation, history of digital amputation, pulmonary hypertension (functional class III-IV) and increased hemorrhagic or thrombotic risk were judged not eligible to NTN in trials.

Results: A total of 177 visits regarding 78 patients were examined (females $80.8 \%$, diffuse skin disease $51.3 \%$, anti-Scl70 antibodies positivity $55.7 \%$, age $54.8 \pm 16.0$ years, disease duration $4.0 \pm 2.4$ years). Considering the visits in which indication for a therapeutic change was given, 54 patients (54.5\%) were eligible for NTN according to SENSCIS criteria and of these 31 (31.3\%) also according to INBUILD criteria (Figure 1). In this group, 25 patients were treated with mycophenolate mofetil, 11 with azathioprine, 10 with cyclophosphamide, 7 with methotrexate and 8 with rituximab ( 2 in combination). At the latest available evaluation, 42 patients $(62.8 \%)$ were eligible for NTN according to SENSCIS criteria and of these $12(15.4 \%)$ also according to INBUILD criteria (Figure 1). In this group, 30 patients were in mycophenolate mofetil ( 6 in combination with biologic treatment), 5 in azathioprine, 1 in cyclophosphamide, 1 in methotrexate, 6 in rituximab, 2 in tocilizumab and 1 in pirfenidone. Overall, the factors limiting NTN start according to the trial enrollment criteria would have been: uncompromised $(19.2 \%)$ or too low $(6.4 \%)$ DLco values, too low FVC (3.4\%), severe acral disease with ulcers $(16.9 \%)$, severe pulmonary arterial hypertension $(6.2 \%)$, increased thrombotic or haemorrhagic risk (6.2\%). In the scenario of eligibility, skin progression was detectable in $43.4 \%$ of all visits.

Conclusion: Treatment that can modify the progression of SSc-ILD are currently limited. Based on our retrospective analysis, the use of NTN in accordance with current clinical evidence could be considered in a significant percentage of patients with SSc-ILD.

REFERENCES:

[1] Flaherty KR, et al. Nintedanib in Progressive Fibrosing Interstitial Lung Diseases. N Engl J Med. 2019

[2] Distler O, et al. Nintedanib for Systemic Sclerosis-Associated Interstitial Lung Disease. N Engl J Med. 2019.

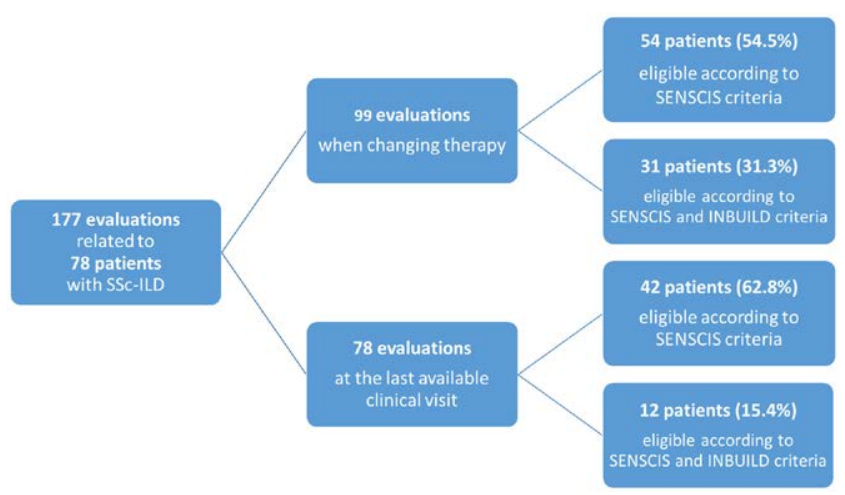

Disclosure of Interests: None declared

DOI: 10.1136/annrheumdis-2021-eular.2711

\section{POS0865 REDUCED BONE MINERAL DENSITY IN PATIENTS WITH IDIOPATHIC INFLAMMATORY MYOPATHIES: A CASE CONTROL STUDY}

Y. K. Tang ${ }^{1}$, V. Lao ${ }^{1}$, H. T. Pang ${ }^{1}$, T. L. V. Wong ${ }^{1}$, T. H. Luk ${ }^{1}$, H. So ${ }^{2} .{ }^{1}$ Kwong Wah Hospital, Medicine and Geriatrics, Hong Kong, Hong Kong (SAR); ${ }^{2} T$ he Chinese University of Hong Kong, Department of Medicine and Therapeutics, Hong Kong, Hong Kong (SAR)

Background: Idiopathic Inflammatory Myopathies (IIMs) patients are at risk of bone mineral density (BMD) loss due to systemic inflammation, use of glucocorticoids (GCs) and disability. Cross sectional study showed $70 \%$ of IIMs patients had reduced BMD but whether they were at excessive risk compared to controls were unknown.

Objectives: To compare the prevalence of reduced BMD between IIMs patients, non-rheumatological controls, rheumatoid arthritis (RA) and systemic lupus erythematosus (SLE) patients and to determine the clinical determinants of BMD in IIMs patients.

Methods: This was a single centre retrospective case control study. BMD at lumbar spine L1-L4 and neck of femur (NOF) were assessed by dual-energy $X$-ray absorptiometry (DXA) scans. The prevalence of reduced BMD and osteoporosis in Chinese IIMs patients and age-and-sex-matched non-rheumatological controls were compared. The BMD of female IIMs were then compared to age matched female RA and SLE patients in the secondary analysis. Binary logistic regression was used for adjustment of confounders. The demographics and 
clinical variables independently associated with BMD were determined by linear regression.

Results: A total of 230 patients were recruited including 65 IIMs, 65 non-rheumatological controls, 50 RA and 50 SLE patients. The mean age was $58.6 \pm 10.96$ years and $76.9 \%$ were female. There was no significant difference on demographics between the two groups. Almost all IIMs patients (98\%) and 52\% of controls had exposed to GCs ( $<<0.001)$. Significantly more IIMs patients had used immunosuppressants (92.3\% vs $38.5 \%, p<0.001)$ and biologics $(13.8 \%$ vs $1.5 \%, p=0.01)$. The prevalence of reduced $\mathrm{BMD}$ and osteoporosis were significantly higher in IIMs patients than non-rheumatological control (Reduced BMD: 73.8\% vs 43.1\%, p=0.043; Osteoporosis: $29.2 \%$ vs $13.8 \%, p=0.033$ ) (Table 1 ). The mean lumbar spine and hip BMD were $0.886 \pm 0.181 \mathrm{~g} / \mathrm{cm}^{2}$ and $0.651 \pm 0.144 \mathrm{~g} / \mathrm{cm}^{2}$ in IIMs patients, which were significantly lower than that of the control group $\left(0.960 \pm 0.143 \mathrm{~g} / \mathrm{cm}^{2}, \mathrm{p}=0.011\right.$ and $0.751 \pm 0.127 \mathrm{~g} /$ $\mathrm{cm}^{2}, \mathrm{p}<0.001$ ) (Figure 1). Multivariate analysis confirmed IIMs were associated with increased risk of reduced BMD (Odds ratio:2.118, $\mathrm{p}=0.048,95 \% \mathrm{Cl} 1.005-4.461$ ). The prevalence of reduced $\mathrm{BMD}$ and osteoporosis were not significantly different between IIMs, RA and SLE patients but the mean hip BMD was the lowest in the IIM group $\left(0.641 \pm 0.152 \mathrm{~g} / \mathrm{cm}^{2}\right.$ vs $0.663 \pm 0.102 \mathrm{~g} / \mathrm{cm}^{2}$ in the RA group vs $0.708 \pm 0.132 \mathrm{~g}$ / $\mathrm{cm}^{2}$ in the SLE group, $\left.\mathrm{p}=0.035\right)$. Lower BMI $(\mathrm{p}=0.035)$ and more advanced age $(p<0.001)$ were associated with lower BMD in the IIM patients.

Conclusion: Reduced BMD was more prevalent in IIM patients than non-rheumatological controls. Lower BMI and more advanced age were associated with lower BMD. Vigilant monitoring of BMD and use of antiresorptive treatment should be considered in IIM patients.

REFERENCES:

[1] Briot K, Geusens P, Em Bultink I et al. Inflammatory diseases and bone fragility. Osteoporos Int. 2017;28:3301-14.

[2] So H, Yip ML, Wong AKM. Prevalence and associated factors of reduced bone mineral density in patients with idiopathic inflammatory myopathies. Int J Rheum Dis. 2016;19:521-8.

Table 1. Prevalence of reduced BMD in IIM patients and non-rheumatologcial controls

\begin{tabular}{llll}
\hline & Myositis $(\mathrm{n}=65)$ & $\begin{array}{l}\text { Non-rheumatological } \\
\text { controls }(\mathrm{n}=65)\end{array}$ & Significance \\
\hline Osteopenia at LS & $25(38.5 \%)$ & $20(30.8 \%)$ & 0.357 \\
Osteoporosis at LS & $13(20 \%)$ & $6(9.2 \%)$ & 0.082 \\
Osteopenia at NOF & $29(44.6 \%)$ & $26(40 \%)$ & 0.542 \\
Osteoporosis at NOF & $12(18.5 \%)$ & $6(9.2 \%)$ & 0.119 \\
Osteopenia overall & $29(44.6 \%)$ & $28(43.1 \%)$ & 0.860 \\
Osteoporosis overall & $19(29.2 \%)$ & $9(13.8 \% \%)$ & 0.033 \\
Reduced BMD overall & $48(73.8 \%)$ & $37(56.9 \%)$ & 0.043 \\
Occurrence of fragility fracture None $=52$ & None=54 & 0.651 \\
& Vertebral $=5$ & Vertebral=4 & \\
& Femoral=1Femoral=1 & Femoral=0 & \\
& Wrist=4 & Wrist $=3$ & \\
& Other sites $=3$ & Other sites=3 & \\
\hline
\end{tabular}

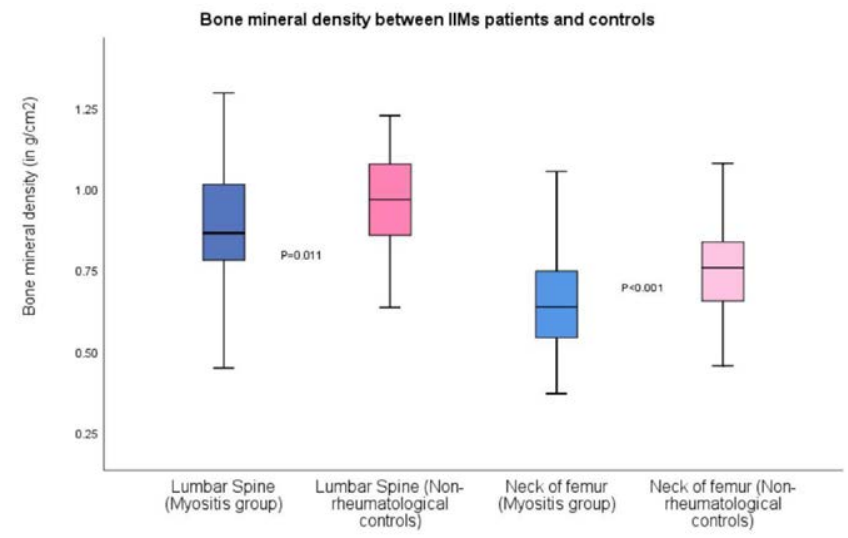

Disclosure of Interests: None declared

DOI: 10.1136/annrheumdis-2021-eular.2824

\section{POS0866 \\ TWO-DIMENSIONAL HRCT-BASED RADIOMIC FEATURES IN SSC-ILD DISTINGUISH DRUG RESPONDERS FROM NON-RESPONDERS}

C. Meier ${ }^{1}$, M. Maciukiewicz ${ }^{1}$, M. Brunner ${ }^{1}$, J. Schniering ${ }^{1}$, H. Gabrys ${ }^{2}$, A. Kühnis ${ }^{2}$, O. Distler ${ }^{1}$, T. Frauenfelder ${ }^{3}$, S. Tanadini-Lang ${ }^{2}$, B. Maurer ${ }^{1}$. ${ }^{1}$ University of Zurich, University Hospital Zurich, Department of Rheumatology, Center of Experimental Rheumatology, Zurich, Switzerland; ${ }^{2}$ University of
Zurich, University Hospital Zurich, Department of Radiation Oncology, Zurich, Switzerland; ${ }^{3}$ University of Zurich, University Hospital Zurich, Institute for Diagnostic and Interventional Radiology, Zurich, Switzerland

Background: Management of patients with systemic sclerosis-associated interstitial lung disease (SSc-ILD) is complicated by high inter-patient variability. To date, no validated predictors of treatment response are available for routine use. High resolution computed tomography (HRCT)-based radiomics, i.e. the high-dimensional, quantitative analysis of imaging metadata, have previously been shown to be successful in discriminating (SSc-)ILD phenotypes in preclinical and clinical studies ${ }^{1}$. Since HRCT is an integral part of the routine work-up in SSc, HRCT-based radiomic features may hold potential as non-invasive biomarkers.

Objectives: To predict treatment response using two-dimensional (2D) HRCT-based radiomics in SSc-ILD patients from a prospectively followed cohort.

Methods: Inclusion criteria were diagnosis of SSc-ILD in HRCT, availability of a suitable chest HRCT scan within 12 months prior to initiation of a new treatment, and availability of clinical baseline and follow-up information. Treatment response was defined as the absence of all of the following over a follow-up period of 12-24 months: relative decrease in forced vital capacity (FVC) $\geq 5 \%$, increase of ILD in $\mathrm{HRCT}$ as assessed by a radiologist, change in treatment regimen due to insufficient response, ILD-related death or lung transplantation. Of each pre-treatment HRCT, 6 slices $(15 \pm 5 \mathrm{~mm}$ apart, starting from the basal lung margin) were manually segmented and $15132 \mathrm{D}$ radiomic features were extracted using the in-house software Z-Rad (Python 2.7). Features were Z-score transformed and pre-filtered for inter- and intra-reader robustness (intraclass correlation coefficient $>0.85$ ) and inter-feature correlation (Spearman's rho <0.9). A categorical linear regression model was created using 3 -fold cross-validated elastic nets for feature selection. Features were then summarized and divided by their number. For generation of a score cut-off, Youden's score was used. For two-group analyses of continuous variables, Wilcoxon's test was performed, whereas categorical data was assessed using Fisher's exact test.

Results: A total of 64 pre-treatment HRCTs from 54 patients were analyzed. In 9 patients, $>1$ asynchronous treatments were assessed, while 45 patients had only 1 eligible treatment approach. The response rate within the assessed follow-up period was $45.3 \%(n=29)$. For score generation, 13 radiomic features were selected and an optimal cut-off value of -0.1589 was determined. Univariate linear regression showed significant association between our categorical radiomics-based score and treatment response $(\mathrm{p}=0.007$, area under the curve $=0.65(0.51-0.79)$, sensitivity $=0.90$, specificity $=0.43)$, whereby a high score was predictive for treatment response.

No differences between patients with high $(n=46)$ or low $(n=18)$ scores were detected for baseline age (mean $\pm S D=55.5 \pm 12.0$ and $55.5 \pm 13.6$ years, $p=0.84$ ), duration of $S S c$ (mean $\pm S D=6.2 \pm 8.4$ and $4.7 \pm 4.4$ years, $p=0.79$ ), time since ILD diagnosis $(2.7 \pm 2.9$ and $2.4 \pm 3.1$ years, $\mathrm{p}=0.59)$, $\mathrm{FVC}(77.6 \pm 20.6$ and $80.1 \pm 17.9$ $\mathrm{p}=0.41)$ or DLco $(54.4 \pm 21.0$ and $57.6 \pm 18.9, \mathrm{p}=0.40)$. Distribution of anti-Scl-70 positivity ( $45.7 \%$ vs. $55.6 \%, p=0.58)$ and diffuse cutaneous disease $(47.7 \%$ vs $61.1 \%, p=0.41$ ) was not significantly different between patients with high and low scores, respectively, although a trend towards higher percentages in the high score group was observed.

Conclusion: Our results indicate that, following validation in external cohorts, radiomics may be a promising tool for future pre-treatment patient stratification. Moreover, our radiomics-based score seems not to be associated with commonly studied clinical predictors such as anti-Scl-70 positivity or lung function, underlining a possible additive value to 'traditional' clinical parameters.

REFERENCES:

[1] Schniering, J., et al. Resolving phenotypic and prognostic differences in interstitial lung disease related to systemic sclerosis by computed tomography-based radiomics. medRxiv [Preprint] doi:10.1101/2020.06.09.20124800 (2020).

Disclosure of Interests: Chantal Meier: None declared, Malgorzata Maciukiewicz: None declared, Matthias Brunner: None declared, Janine Schniering: None declared, Hubert Gabrys: None declared, Anja Kühnis: None declared, Oliver Distler Speakers bureau: Speaker fee on Scleroderma and related complications: Bayer, Boehringer Ingelheim, Medscape, Novartis, Roche. Speaker fee on rheumatology topic other than Scleroderma: MSD, iQone, Novartis, Pfizer, Roche, Consultant of: Consultancy fee for Scleroderma and its complications: Abbvie, Acceleron Pharma, Amgen, AnaMar, Arxx Therapeutics, Bayer, Baecon Discovery, Boehringer, CSL Behring, ChemomAb, Corbus Pharmaceuticals, Horizon Pharmaceuticals, Galapagos NV, GSK, Glenmark Pharmaceuticals, Inventiva, Italfarmaco, iQvia, Kymera, Medac, Medscape, Mitsubishi Tanabe Pharma, MSD, Roche, Roivant Sciences, Sanofi, UCB. Consultancy fee for rheumatology topic other than Scleroderma: Abbvie, Amgen, Lilly, Pfizer, Grant/research support from: Research Grants to investigate the pathophysiology and potential treatment 